\title{
Twenty-four-hour Blood Pressure Monitoring in Central Serous Chorioretinopathy - A Case-control Study
}

\author{
Pradeep Venkatesh, ${ }^{1}$ Vikas Jain ${ }^{2}$ and Satpal Garg ${ }^{3}$
}

1. Additional Professor, Retina Services; 2. Resident; 3. Professor, Retina Services, Dr Rajendra Prasad Centre for Ophthalmic Sciences, All India Institute of Medical Sciences, New Delhi, India

\section{Abstract}

Purpose: A case-control study to explore the diurnal variation of blood pressure (BP) in patients with central serous chorioretinopathy (CSCR). Methods: One-hundred patients ( 50 cases with central serous chorioretinopathy and 50 age- and sex-matched controls) participated in this study. Twenty-four-hour BP was recorded by auscultatory method using manual standard mercury sphygmomanometer by a single, trained observer. Hypertension was categorised according to the Seventh Report of the Joint National Committee on Prevention, Detection, Evaluation, and Treatment of High Blood Pressure (JNC-VII) classification. Results: Mean systolic BP (SBP) and diastolic BP (DBP) in cases and controls were $123.4 \mathrm{mmHg}$ (range 112.3-134.6), $81.72 \mathrm{mmHg}$ (range 73.0-90.5); $118.33 \mathrm{mmHg}$ (range 108.4-128.2); and 77.50 mmHg (range 71.4-83.6), respectively. The difference in SBP and DBP was statistically significant at $p=0.02$ for the former and $p=0.006$ for the latter. The mean difference in maximum and minimum SBP in cases was $18( \pm 15.81) \mathrm{mmHg}$ versus $9.04( \pm 3.08) \mathrm{mmHg}$ in controls ( $p=0.001)$. Mean difference for DBP in cases was $15.16( \pm 7.86) \mathrm{mmHg}$ versus $9.16( \pm 3.68) \mathrm{mmHg}$ in controls $(0.001)$. On the JNC-VII classification scale for hypertension, $66 \%$ of cases were found to be pre-hypertensive $(60 \%)$ or hypertensive $(6 \%)$. Comparative figures for controls were pre-hypertension (30\%) and hypertension (2\%). The mean SBP and DBP was consistently higher in patients with CSCR compared with controls over the entire 24-hour period. The difference in variation between maximum and minimum SBP and DBP was also found to be statistically significant between the groups. Conclusion: A significant correlation exists between higher values BP and central serous chorioretinopathy. Screening of CSCR patients for pre-hypertension may be useful in decreasing the risk of progression to established hypertension, with its associated risks.
\end{abstract}

\section{Keywords}

Blood pressure, hypertension, central serous retinopathy, case-control study

Disclosure: The authors have no conflicts of interest to declare.

Received: 20 October 2012 Accepted: 14 March 2013 Citation: European Ophthalmic Review, 2013;7(1):42-4 DOl: 10.17925/EOR.2013.07.01.42

Correspondence: Pradeep Venkatesh, E-104, AllMS campus, Ansari Nagar (East), New Delhi-110029, India. E: venkyprao@yahoo.com

Hypertension has previously been reported as a possible risk factor for the development of central serous chorioretinopathy (CSCR). In these studies, hypertension was documented retrospectively and based on patient questionnaires or as an incidental finding during the evaluation of sympathetic-parasympathetic nervous system in patients with central serous retinopathy. ${ }^{1-3}$ We report herein, for the first time, results of a prospective, case-control study that was designed to address the role of hypertension in CSCR by determining its diurnal variation and comparing with a control group.

\section{Methods}

A total of 100 patients (50 cases with CSCR and 50 age- and sexmatched controls) participated in this study. The study was approved by the Institute Ethics committee, and each subject provided Informed consent before the start of the study. All cases had clinical features of CSCR and this was confirmed by the documentation of active leakage on fluorescein angiography. All patients presented with the first episode and the duration of symptoms ranged from 1 week to 6 weeks. None of these patients had received any treatment prior to enrolment. Those wrongly being treated with corticosteroids prescribed elsewhere were not included in the study. Young adults admitted for vitreoretinal surgery (unilateral retinal detachment or idiopathic vitreous haemorrhage) were chosen as controls. The reason for choosing young patients with the above pathologies as controls was twofold. First, to have a group of patients who were about the same age group in which CSCR tends to occur. Second, to address the possible role of stress-related blood pressure (BP) changes (due to unilateral loss of vision and also from hospital admission 'white coat hypertension'). Patients with vitreous haemorrhage included in this study did not have any systemic ailment that could have affected the BP. In both groups, patients with known hypertension were not enrolled into the study. No BP evaluation was performed prior to admitting the patients for the study and all measurements were prospectively undertaken.

BP was recorded by auscultatory method using standard mercury sphygmomanometer by a single, trained observer (VJ). Subjects were advised to rest for at least 15 minutes before each BP recording and refrain from taking any food or beverages for 2 hours prior to the test. BP was measured at 3 hourly intervals in the right upper arm starting from 10.00 AM to 7.00 AM the next morning. Two readings at 3 minute intervals were taken at each recording, with the patient in supine position and using an appropriately sized cuff. Korotkoff phase 1 was considered as systolic BP (SBP) and phase 5 as diastolic BP (DBP). 
Hypertension was categorised according to the Seventh Report of the Joint National Committee on Prevention, Detection, Evaluation, and Treatment of High Blood Pressure (JNC-VII) classification.

The data was recorded on an Excel worksheet and was analysed using SPSS 13.0 software. The groups were compared using an independent t-test. For this study, $p$ value was taken as two-tailed. $p$ values less than 0.05 was considered statistically significant.

Stress (subjective) was based on selected negative life events - loss of mother/father/sibling/close relatives/friends, unemployment/loss of job, poor performance in studies, unpleasant relationship in family, etc. Global anxiety, depression, and mental stress were assessed by the following questions: "Do you think you are anxious or tense or have worries?" "Do you feel depressed, low, and blue?" and "Do you feel under mental stress?"

\section{Results}

Mean age of subjects in the cases (35.36 $[ \pm 5.95]$ years) and in controls (35.76 $[ \pm 6.00]$ years)] was similar. There were three female patients in each group. Mean SBP and DBP in cases and controls was $123.4 \mathrm{mmHg}$ (range 112.3-134.6); $81.72 \mathrm{mmHg}$ (range 73.0-90.5) and $118.33 \mathrm{mmHg}$ (range 108.4-128.2); $77.50 \mathrm{mmHg}$ (range 71.4-83.6), respectively. The difference in SBP and DBP was statistically significant at $p=0.02$ for the former and $\mathrm{p}=0.006$ for the latter. On the JNC-VII classification scale for hypertension, $66 \%$ of cases were found to be pre-hypertensive (60\%) or hypertensive (6\%). Comparative figures for controls were prehypertension (30\%) and hypertension (2\%).

Diurnal variation of the SBP and DBP between the two groups is shown in Figure 1 and Figure 2. The mean SBP and DBP was consistently higher in patients with CSCR compared with controls over the entire 24-hour period. For SBP, the difference between the groups was found to be statistically significant for recordings at $10.00 \mathrm{AM}(\mathrm{p}=0.042), 1.00 \mathrm{PM}$ $(p=0.005), 4.00 \mathrm{PM}(\mathrm{p}=0.015)$ and $10.00 \mathrm{PM}(\mathrm{p}=0.004)$. For diastolic pressure, a statistically significant difference was noted at $1.00 \mathrm{PM}$ $(p=0.01), 4.00$ PM $(p=0.009), 7.00$ PM $(p=0.002), 10.00$ PM $(p=0.001)$ and $1.00 \mathrm{AM}(\mathrm{p}=0.05)$.

The difference in variation between maximum and minimum SBP and DBP was also found to be statistically significant between the groups. Mean difference for SBP in cases was $18( \pm 15.81) \mathrm{mmHg}$ vs $9.04( \pm 3.08)$ $\mathrm{mmHg}$ in controls $(\mathrm{p}=0.001)$. Mean difference for DBP in cases was $15.16( \pm 7.86) \mathrm{mmHg}$ versus $9.16( \pm 3.68) \mathrm{mmHg}$ in controls $(p=0.001)$.

On determining the odds ratio (OR), patients with raised SBP ( $\geq 120 \mathrm{mmHg}$ ) were 4.13 times more prone to develop CSCR than patients with normal SBP $(<120 \mathrm{mmHg})$ and those with raised DBP $(\geq 80 \mathrm{mmHg}$ ) were 3.27 times more prone to develop CSCR than patients with normal DBP ( $<80 \mathrm{mmHg})$.

\section{Discussion}

Several systemic associations have also been reported earlier in patients with CSCR. ${ }^{1-3}$ The most widely accepted risk factors have been type A personality and stress. ${ }^{4}$ Tittl et al. in 1999 in their retrospective study identified psychopharmacological medication use, corticosteroid use and hypertension as factors associated with CSCR. ${ }^{1}$ Haimovici et al., in 2003, in their retrospective case control study found that previously described risk factors, systemic steroid use (OR 37.1 $95 \%$ confidence interval [Cl] 6.2-221.8) and pregnancy (OR $7.195 \% \mathrm{Cl}$

\section{Figure 1: Diurnal Variation of Systolic Blood Pressure}

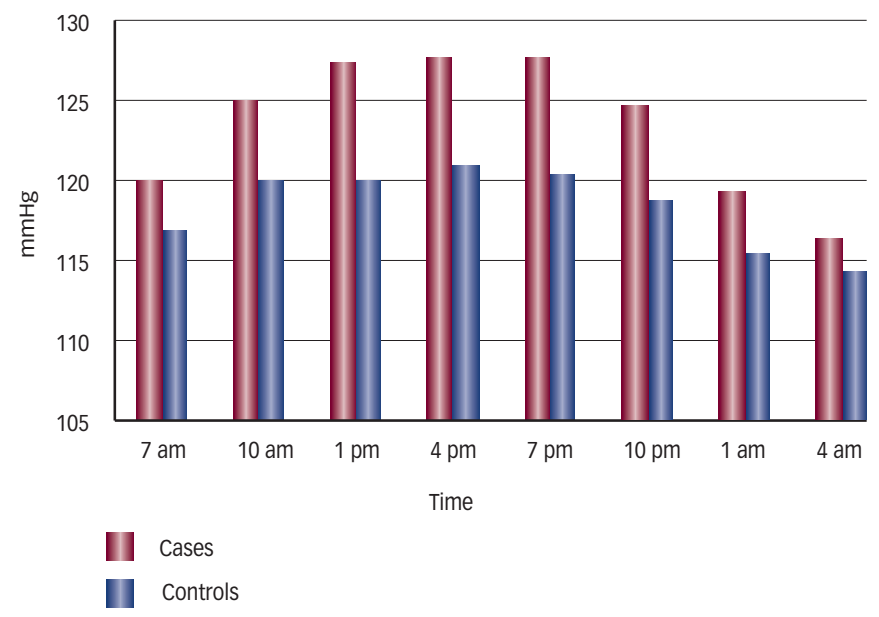

\section{Figure 2: Diurnal Variation of Diastolic Blood Pressure}

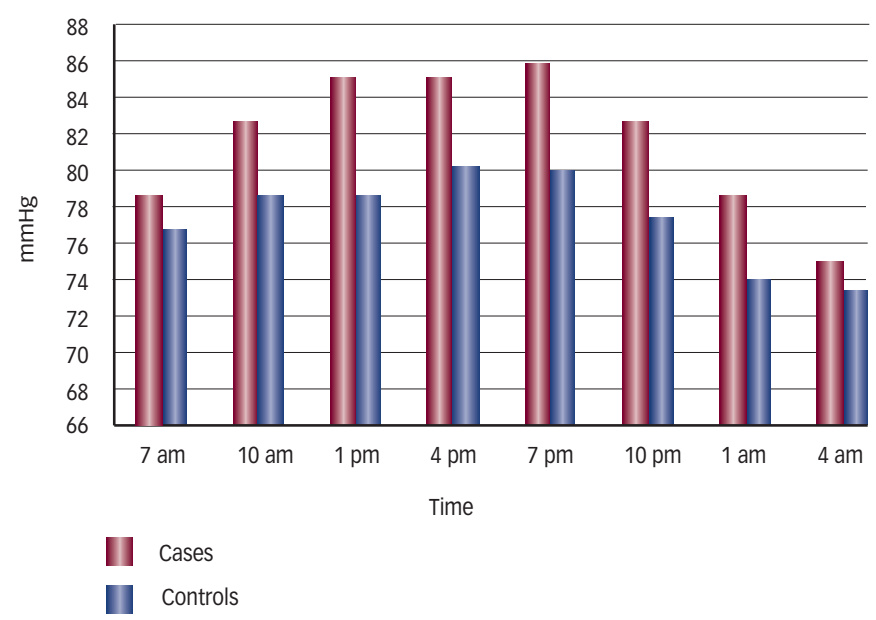

1.0-50.7), were strongly associated with CSCR. Additional risk factors identified by this study included antibiotic use (OR $6.295 \% \mathrm{Cl} 1.0$ 37.9), alcohol use (OR $4.995 \% \mathrm{Cl} 1.5-16.3)$, untreated hypertension (OR $3.395 \%$ Cl 1.3-8.5), an allergic respiratory disease (OR $2.595 \%$ Cl 1.2-5.1). ${ }^{2}$

A limitation of these studies was that results were based on patient questionnaire data with no actual clinical evaluation of BP. Contrary to these reported publications, the observations in our study are based on measurement of BP over a 24-hour period in a prospective manner. Our results add further strength to the possible association between CSCR and systemic hypertension.

It is possible that the pathway for neurosensory detachment to occur in these patients is mediated by BP regulation instability. Being modifiable, stabilisation of BP towards lower levels may also aid in reducing the risk of recurrence, reported to occur in about $45 \%$ of patients. ${ }^{5}$

Identification of elevated BP as a risk factor in patients with central serous retinopathy is important for another reason. Hypertension is the commonest cause for overall morbidity and mortality in the elderly population. ${ }^{6}$ Pre-hypertension is known to progress to higher grades 


\section{Retina}

of hypertension. This natural progression can be effectively retarded as BP is modifiable by early lifestyle and dietary interventions.

An important limitation of this study was our inability to perform a 24hour automated BP monitoring due to the high cost and lack of such facility at our institute.

\section{Conclusion}

The results of this study suggest that a statistically significant correlation exists between elevated BP, its diurnal variation and CSCR. To the best of our knowledge, this is the strongest evidence of the association reported to date. Hence, regular screening of patients with CSCR for hypertension may be useful follow up advice.
1. Tittl MK, Spaide RF, Wong D, et al., Systemic findings associated with central serous chorioretinopathy,

Am J Ophthalmol, 1999;128:63-8.

2. Haimovici R, Koh S, Gagnon DR, et al., Risk factors for central serous chorioretinopathy: a case-control study, Ophthalmology, 2004;111:244-9.
3. Venkatesh P, Gadia R, Tewari HK, et al., Prehypertension may be common in patients with central serous chorioretinopathy, Graefes Arch Clin Exp Ophthalmol, 2006;244(9):1101-3. Yannuzzi LA, Type-A behavior and central serous chorioretinopathy, Retina, 1987;7(2):111-31

5. Klein ML, Van Buskirk EM, Freidman E, et al., Experience with non-treatment of central serous choroidopathy, Arch Ophthalmol, 1974;91:247-50.

Chobanian AV, Bakris GL, Black HR, et al., The seventh report of the Joint National Committee on Prevention, Detection, Evaluation, and Treatment of High Blood Pressure: the JNC 7 report, JAMA, 2003;289:2506-72. 